\title{
Digitalisierte Gesundheit: neue Herausforderungen für Public Health
}

\author{
Digital Health: new Challenges for Public Health
}

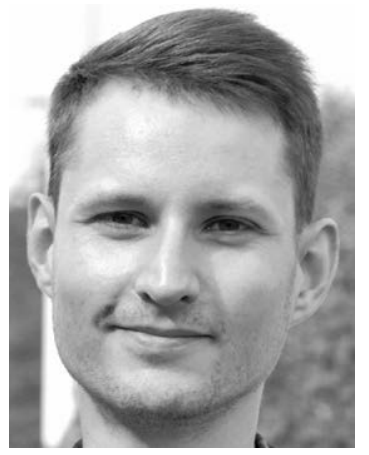

C. Dockweiler

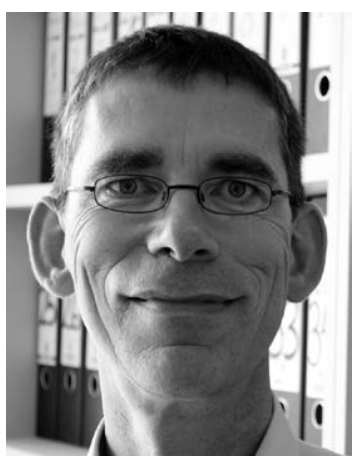

O. Razum
E-Health erfährt aktuell eine bemerkenswerte Aufmerksamkeit. In Europa hat hierzu die strategische Förderung der Europäischen Union (EU) im Rahmen des „eHealth Action Plan 2012-2020“ beigetragen [1]. Die nationale gesundheitspolitische Diskussion war dagegen durch eine fragmentierte und wenig systematische Road-Map mit fehlendem Mut zu einer klaren strukturell-politischen Rahmung der Entwicklung von Informations- und Kommunikationstechnologien im Gesundheitswesen geprägt. Das betraf nicht nur finanzierungsbezogene, berufs- und datenschutzrechtliche Aspekte. Auch die Förderung von Versorgungs-, Interventions- und Technikfolgeforschung sowie die Aus-, Fort- und Weiterbildung wurden stiefmütterlich behandelt. Die deutsche Public Health sah diese Entwicklung überwiegend kritisch, auch wegen der fehlenden Evidenz für die neuen Versorgungstechnologien oder den wirtschaftlichen Interessen der Förderung [2].

Anfang 2016 soll durch das Gesetz für sichere digitale Kommunikation und Anwendungen im Gesundheitswesen (das sogenannte „E-HealthGesetz“) der Grundstein für eine konzertierte Entwicklung und Implementation von E-Health gelegt werden. Der Einsatz von Informationsund Kommunikationstechnologien im Gesundheitswesen bietet Chancen für Patientinnen und Patienten, bringt aber auch Probleme mit sich. Die interdisziplinäre Perspektive von Public Health kann im E-Health-Diskurs gewinnbringend zwischen technologischen, individualmedizinischen und bevölkerungsbezogenen Sichtweisen E-Health als systematisierte und anwendungsbezogene Form von Digitalisierungsprozessen im Gesundheitswesen die Perspektive von Public Health und die Implementierung von PublicHealth-Interventionen bereichern. Im vorliegenden Beitrag zeigen wir fünf Herausforderungen und Chancen auf und möchten damit zur Klärung der Beziehung zwischen E-Health und Public Health beitragen.

Online-Publikation: 22.12.2015

Gesundheitswesen 2016; 78:

5-7 @ Georg Thieme

Verlag KG Stuttgart · New York ISSN 0941-3790

Korrespondenzadresse

Christoph Dockweiler

Fakultät für Gesundheitswissenschaften

Universität Bielefeld

Universitätsstraße 25

33615 Bielefeld

christoph.dockweiler@uni-

bielefeld.de

Erste Herausforderung: begriffliche Klarheit

$\nabla$

Bislang fehlt eine einheitliche Definition von E-Health. Die Anwendungsfelder von E-Health, Gesundheitstelematik oder Telemedizin sind unscharf eingegrenzt und überlappend [3]. EHealth kann als verbindendes Element verstanden werden, unter dem jeglicher Einsatz von Kommunikations- und Informationstechnologien und Interessen vermitteln. Gleichzeitig könnte

(ICT) im Bereich Gesundheit und Krankheit verstanden wird. Im deutschsprachigen Raum hat sich parallel dazu der Begriff der Gesundheitstelematik etabliert, welcher häufig synonym verwendet wird. E-Health-Leistungen lassen sich in Anlehnung an Kacher et al. [4] innerhalb von 5 Gestaltungsbereichen (Inhalt, Ökonomie, Vernetzung, Gesundheit, Versorgung) strukturieren - Abb. 1.

Die Abgrenzung der Ebenen, innerhalb derer E-Health wirksam werden kann, ist jedoch keineswegs trennscharf. Vielmehr findet in der praktischen Umsetzung häufig eine Synthese verschiedener Leistungen und der hier zugehörigen Datensätzen statt.

\section{Zweite Herausforderung: Autonomie oder Kontrolle? \\ $\nabla$}

Die Entwicklung von E-Health war meist Thema einer disziplinären, überwiegend technik- und/ oder medizindominierten Diskussion. Fragen einer bedarfsgerechten Umsetzung und Nutzung von Gesundheitstechnologien lassen sich nicht allein durch die Prämisse des „technisch Möglichen“ lösen, sondern müssen sich am „technisch Nötigen“ orientieren. Durch technische Fortentwicklung erweitert sich das Repertoire der Interventionsmöglichkeiten so schnell, dass Diskussionen über deren soziale und gesundheitsbezogene Folgen oft erst im Nachgang geführt werden. Das betrifft besonders den schmalen Grat zwischen Autonomie und externer Kontrolle bei der automatisierten Erfassung von Gesundheitsdaten. Diese findet nicht nur im Rahmen von medizinischer und pflegerischer Versorgung statt, sondern neuerdings in großem Maßstab auf dem Consumer-Health-Market. Die automatisierte Datenerfassung (z.B. über Implantate, Kameras, Sensorik in der häuslichen Umgebung oder in der Kleidung) steigert zwar das Gefühl, gesundheitsrelevante Vorgänge unter Kontrolle zu haben. Eine Emanzipation der Nutzerinnen und Nutzer bzw. Patientinnen und Patienten bleibt dennoch fraglich. 


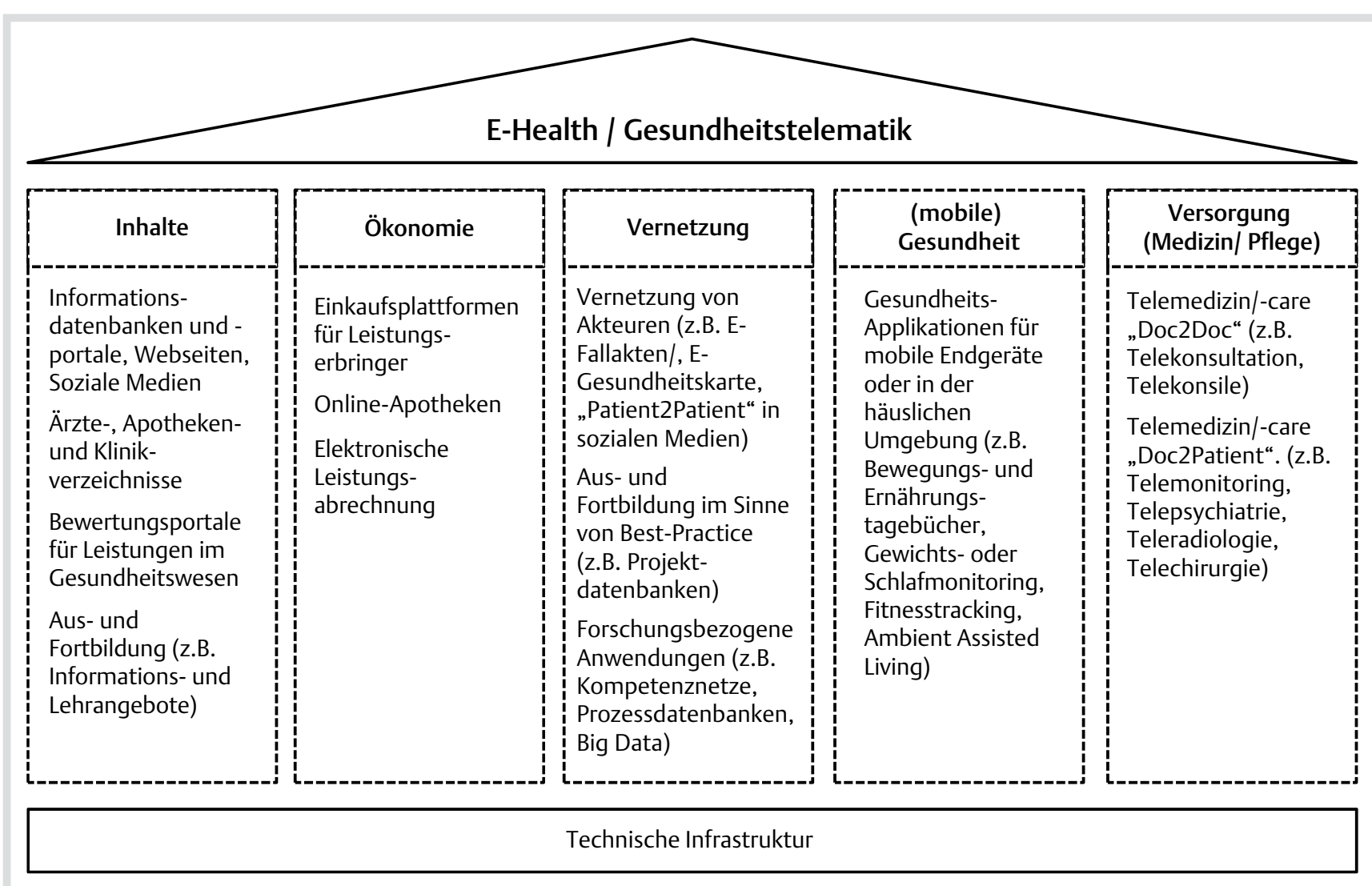

Abb. 1 Strukturierung von E-Health-Leistungen.

außerhalb des Medizinsystems agieren. Zum einen können dies Konzerne wie Google oder Apple sein, die über Smartphones Daten bspw. zum Bewegungsverhalten ihrer Kunden sammeln und diese mit Daten über Konsumgewohnheiten kombinieren können. „Big Data“, in der Wissenschaft noch kritisch hinsichtlich möglicher Erkenntnisgewinne und offensichtlicher Datenschutzprobleme diskutiert, ist hier längst Wirklichkeit. Zum anderen könnten Krankenversicherungen in Versuchung kommen, durch Gesundheitsverhaltensanalysen bei ihren Versicherten zwischen „guten“ und „schlechten“ Risiken zu unterscheiden. Autonomie und Kontrolle müssen zukünftig also auch im Sinne von „Informationstransparenz“ verstanden werden. Dies muss sich auch das Medizinsystem zu eigen machen - Patientinnen und Patienten werden wissen wollen, ob ihre vertraulichen Gesundheitsinformationen innerhalb der medizinischen und pflegerischen Versorgungskontexte sicher aufgehoben sind. Allein der Verdacht, dass unberechtigte Zugriffe erfolgen könnten, würde schnell zu einem Vertrauensverlust führen. Die informationelle Selbstbestimmung ist daher zentraler Baustein der Datenverarbeitung im Gesundheitswesen und der Diskussion um „Big Data“ - dies wurde und wird auch gesundheitspolitisch erkannt und zumindest sukzessive umgesetzt. Doch sind die Nutzerinnen und Nutzer in der Lage, diese Selbstbestimmung auch im Alltag umzusetzen?

\section{Dritte Herausforderung: neue Technologien und alte Ungleichheiten?}

Soziale Ungleichheit geht mit gesundheitlicher Ungleichheit einher, das ist eine der Grunderkenntnisse von Public Health.
Soziale Ungleichheit ist aber auch mit digitaler Ungleichheit assoziiert. E-Health soll eigentlich dazu beitragen, Versorgungsungleichheiten zu dämpfen - etwa räumliche Ungleichheiten mithilfe der Telemedizin. Die Technisierung könnte jedoch neue Ungleichheiten im Zugang zu und in der Nutzung von E-Health selbst schaffen. Digitale Ungleichheit kann zu sozialer und letztendlich auch gesundheitlicher Ungleichheit führen, wenn aus der fehlenden Verfügbarkeit neuer Technologien Einschränkungen von Lebenschancen resultieren - bspw. durch geringeres Wissen über die eigenen Gesundheit und Krankheit, über Versorgungsoptionen oder alternative Therapie- und Versorgungsansätze. Ungleichheiten im Zugang zu E-Health könnten dabei mit dem sozioökonomischen Status, dem kulturellen Hintergrund, Gender, Alter oder dem Wohnort zusammenhängen [5]. Auf europäischer Ebene werden die Ziele von E-Health bereits vor diesem Hintergrund diskutiert, sie beinhalten explizit „socio-economic inclusion and equality, quality of life and patient empowerment through greater transparency, access to services and information and the use of social media for health" ([1]: S. 5.) Hieran muss die Diskussion in Deutschland noch Anschluss finden.

\section{Vierte Herausforderung: Nutzerinnen- und Nutzer- orientierung \\ $\nabla$}

In Deutschland ist der E-Heath-Diskurs stark mit einer individualisierten und technikzentrierten Medizin (und immer mehr auch Pflege) verknüpft. Das zeigen Sammelbände wie z.B. der von Jähn und Nagel [6], aber auch die aktuelle Gesetzgebung. Die Diskussion technischer Aspekte ist zwar ein notwendiger 
Bestandteil der gesellschaftlichen Diffusion von Technologien, und natürlich muss auch eine angemessene (und standardisierte) Technikinfrastruktur geschaffen werden. Doch erscheint diese Engführung bisher mehr als Diskussion „über“ als „mit“ den Nutzerinnen und Nutzern. Nur wenige Beiträge betrachten das breite Geflecht der Bedarfe, Einstellungen, Wertehaltungen, Sorgen oder Vorbehalte, die in den unterschiedlichen Nutzerinneund Nutzergruppen vorliegen (kurzum: Nur wenige Beiträge nehmen eine Public-Health-Perspektive ein). Dabei ist der Diskurs „mit“ den Nutzerinnen- und Nutzern unverzichtbar - nicht nur mit Blick auf die Akzeptanz, sondern auch hinsichtlich der zielgruppenspezifischen und bedarfsgerechten Ausgestaltung von E-Health.

Public Health muss hier zu einer partizipativen Versorgungsgestaltung beitragen. Dabei sind E-Health-Leistungen auch auf ihre gesellschaftliche Wirkung (auf unterschiedliche Bevölkerungsgruppen und das Gesundheitssystem) hin zu prüfen. Hierfür bedarf es jedoch Forschungsförderstrukturen, die unabhängig von wirtschaftlichen Interessen, den Blick von der technischen Entwicklung lösen und auf die Wirkung, Wahrnehmung und die Nutzung neuer Informations- und Kommunikationstechnologien im Gesundheitswesen richten.

\section{Fünfte Herausforderung: Chancen für Public Health erkennen}

Technologische Innovationen wirken (re-)strukturierend auf soziale Zusammenhänge - sie sind somit gleichzeitig soziale Innovationen [7]. Die Auseinandersetzung mit E-Health bietet daher Querbezüge und Potenziale für den Public-Health-Diskurs. So ermöglichen neue Technologien neue Interaktionsformen in der Gesellschaft und in ihren Teilbereichen wie dem Gesundheitssystem. Dies zeigt sich nicht nur in neuen Weisen, wie Menschen Gesundheitsinformationen suchen, rezipieren und wiederum selber innerhalb von Blogs, Foren und anderen sozialen Medien produzieren [8]. Es zeigt sich auch darin, wie soziale Online-Netzwerke sich auf die soziale und psychische Gesundheit auswirken [9] und wie Krankheiten bewältigt werden [10]. Schlussendlich zeigt es sich darin, wie gesundheitliche Versorgung technikunterstützt gestaltet [11] und wie durch (neue) Medien Angebote sowie Botschaften der Prävention nachhaltig und zielgruppenspezifisch in die Lebenswelten integriert werden [12].

Eine weitere, schwerer einzuschätzende Chance liegt im Bereich der „Big Data“. Datensammlungen und -analysen ermöglichen tiefe Einblicke in den Lebenswandel und die gesundheitlichen Risiken unterschiedlicher Bevölkerungsgruppen, und das zeitnah und mit wiederholten Messungen - Epidemiologinnen und Epidemiologen können hiervon oft nur träumen. „Big Data“ bringen zugleich aber auch ethische und rechtliche Herausforderungen mit sich: Probleme bei der Dateninterpretation, Datenmissbrauch mit Vertrauensverlust gegenüber der Forschung oder gar eine digitale Bevormundung.

\section{Fazit}

Vielleicht wird eine wirklich nutzerinnen- und nutzerorientierte, demokratische und den einzelnen Menschen achtende E-Health ein Traum bleiben. Sollte sie aber Wirklichkeit werden, so hätte sie aus Sicht von Public Health große Potenziale, sogar hinsichtlich größerer Chancengleichheit und Empowerment. E-Health könnte etwa durch die Vernetzung unterschiedlicher Leistungserbringer auf allen Versorgungsebenen dazu beitragen, in Gebieten mit geringer medizinischer Infrastrukturdichte die Versorgungssicherheit und -qualität zu gewährleisten. Sie könnte Gesundheitsinformationen und Informationen zu Rechtsansprüchen bspw. bei Krankheit niedrigschwellig über mobile Endgeräte bereitstellen. Und sie könnte Bürgerinnen und Bürger vermehrt über gesundheitsrelevante Einflüsse der Lebensumwelt und des eigenen Verhaltens aufklären und in Entscheidungen bezüglich Prävention, Gesundheitsförderung und medizinisch-pflegerischer Versorgung einbeziehen.

Eine Voraussetzung dafür, dass dieser Traum Wirklichkeit wird und E-Health seine positiven Potenziale entfalten kann, ist die industrieunabhängige Förderung einer kritischen Begleitforschung. Hierin liegt eine weitere Herausforderung und Chance für Public Health: Sie muss in der häufig technikgetriebenen Entwicklung von E-Health einen Perspektivwechsel hin zu einer konsequenten Nutzerinnen- und Nutzerorientierung einleiten, technologische Innovationen mithilfe relevanter gesundheitlicher Endpunkte evaluativ begleiten und Technikfolgen in sozialer, pflegerischer und gesundheitlicher Hinsicht interdisziplinär beleuchten. Public Health ist für E-Health unentbehrlich.

Interessenkonflikt: Die Autoren geben an, dass kein Interessenkonflikt besteht.

\section{Literatur}

1 European Commission. eHealth Action Plan 2012-2020 - Innovative healthcare for the $21^{\text {st }}$ century. http://ec.europa.eu/information society/newsroom/cf/dae/document.cfm?doc_id=4188 Gesehen am 20.07.2015

2 Braun B. Nur „cash cow“ oder doch nützlich für Patienten? Die EU-Kommission und das „Lied vom großen Nutzen“ von E-Health. http://www.forum-gesundheitspolitik.de/artikel/artikel.pl?artikel = 1930 Gesehen am 20.07.2015

3 Häckl D. Medizinisch-technischer Fortschritt, e-Health und Telemedizin. Wiesbaden: Gabler; 2010

4 Kacher C, Wiest A, Schumacher N. E-Health: Chancen und Risiken für Ärzte, Patienten und Kostenträger. Zeitschrift für Allgemeinmedizin 2000; 76: 607-613

5 Marschang $S$. Health inequalities and eHealth. European Commission http://ec.europa.eu/information_society/newsroom/cf/dae/document. cfm?doc_id $=5170$ Gesehen am 20.07.2015

6 Jähn K, Nagel E., (Hrsg.). e-Heath. Berlin: Springer; 2004

7 Rogers EM. Diffusion of Innovations. 5. Auflage. New York: Free Press; 2003

8 Rossmann C, Karnowski V. eHealth und mHealth: Gesundheitskommunikation online und mobil. In: Hurrelmann K, Baumann E, (Hrsg.). Handbuch Gesundheitskommunikation. Bern: Hans Huber; 2015: 271-285

9 Kneidinger B. Facebook und Co. Eine soziologische Analyse von interaktionsformen in Online Social Networks. Wiesbaden: VS; 2010

10 Best P, Manktelow R, Taylor B. Online communication, social media and adolescent wellbeing: A systematic narrative review. Children and Youth Services Review 2014; 41: 27-36

11 Van den Berg N, Schmidt S, Stenzel U et al. Telemedizinische Versorgungskonzepte in der regionalen Versorgung ländlicher Gebiete. Bundesgesundheitsblatt 2015; 58: 367-373

12 Lupton $D$. Health promotion in the digital era: a critical commentary. Health Promotion International 2014; 30: 174-183 